\title{
Color Flavor Locked Phase Transition in Strange Quark Matter
}

\author{
Milva Orsaria ${ }^{a}$, H. Rodrigues ${ }^{b}$, and S. B. Duarte ${ }^{a}$ \\ ${ }^{a}$ Centro Brasileiro de Pesquisas Físicas \\ Rua Dr. Xavier Sigaud 150 CEP 22290-180, Rio de Janeiro, Brazil \\ ${ }^{b}$ Centro Federal de Educação Tecnológica CEFET/RJ, \\ CEP 20271-110, Rio de Janeiro, Brazil
}

Received on 29 September, 2006

\begin{abstract}
We discuss macroscopic aspects of quark matter phase transition in cold dense stellar matter, considering global charge neutrality and baryonic charge conservation. We determine the critical condition for the phase transition between the strange quark matter, $S Q M$, and the color-flavor locked, $C F L$, superconducting phase. We also discuss the sensitivity of our results to variations in the gap energy, $\Delta$, and in the current strange quark mass, $m_{s 0}$. The phase transition is calculated taking into account the baryonic density dependence of the quark masses in dense baryonic medium.

Keywords: Cold dense stellar matter; Phase transition; Color superconductivity
\end{abstract}

\section{INTRODUCTION}

The strange quark matter $(S Q M)$ is supposed to be the most stable quantum state of the hadronic matter [1],[2]. The $S Q M$ can be originated by a first-order hadron-quark phase transition occurring in the core of a massive star at the end of its evolutionary path when a gravitational collapse of the structure takes place. This hadron-quark phase transition leads to a deconfined quark gas at densities presumably near to 2-3 times the normal nuclear matter density. At sufficiently large baryon chemical potential, the more stable configuration of $S Q M$ is the color flavor locked $(C F L)[3]$ superconducting phase in which Cooper pairs of quarks of different flavors and colors are coupled with total zero momentum. For very high density, the mass of the strange quark is negligible compared to the baryonic chemical potential, leading to the same density of the three flavors of $u, d$ and $s$ quarks. Consequently, the $C F L$ phase is naturally electrically neutral.

In the present work we study the phase transition between the $S Q M$ and $C F L$ phases, determining the critical value of the baryonic chemical potential, $\mu$, for different baryonic densities. A phenomenological model taking into account the dependence of the quark mass with the baryonic density is employed: the dynamical density quark mass $D D Q M$ model[4]. The sensitivity of the calculated values for the critical chemical potential for the $S Q M-C F L$ phase transition is analyzed in respect to the parameter of $D D Q M$ model used. We also discuss some results obtained when the values of the color superconducting gap and the current strange quark mass are both changed.

\section{THE $D D Q M$ MODEL IN THE $S Q M$ PHASE}

According to the low-density regime of $Q C D$, the quark masses are not merely originated from the explicit breaking of chiral symmetry. So, in the study of stability of quark matter, we can not neglect the dynamical mass induced by the strong quark interaction with the medium. For low and moderate density regime, we model the dynamical mass by the density dependent quark mass given by[4], [5]

$$
M_{D}=\frac{C}{3 n_{B}},
$$

where $n_{B}$ denotes the baryon density and $C$ is a free parameter that in the zero density limit $\left(n_{B} \rightarrow 0\right)$ correspond to the constant energy density in the $D D Q M$ model. For null current masses of quarks $u$ and $d$ and for the current strange quark mass, $m_{s 0}$, we have

$$
\begin{aligned}
& m_{u}^{*}=m_{d}^{*}=M_{D}, \\
& m_{s}^{*}=m_{s 0}+M_{D} .
\end{aligned}
$$

The $D D Q M$ model establish the effective quark mass values in pertubative and nonperturbative regime through the dependence with the dynamical mass $M_{D}$. With this model two characteristic situations for quark matter (the confinement of quarks and asymptotic freedom regime)is reproduced when the corresponing densities regime is considered.

We consider the quark matter in stellar medium, that can be treated as a degenerate Fermi gas of $u, d, s$ quarks and electrons with chemical equilibrium maintained by the weak interaction processes

$$
\begin{aligned}
d(s) & \longleftrightarrow u+e^{-}+\overline{\mathrm{v}}_{e} \\
u+d & \longleftrightarrow u+s .
\end{aligned}
$$

If neutrinos freely scape from the medium, playing no role on the beta equilibrium conditions, the processes displayed above imply the following relation between the chemical potentials:

$$
\begin{gathered}
\mu_{u}=\mu_{d}-\mu_{e}, \\
\mu_{d}=\mu_{s} .
\end{gathered}
$$

leaving only two independent chemical potentials for the thermodynamical description of the system. 
When the temperature is small enough, the antiquarks are statistically negligible and the density of fermions in the absence of interactions may be approximated by

$$
\frac{N_{q}}{V}=n_{q} \simeq \frac{1}{\pi^{2}}\left(\mu_{q}^{2}-m_{q}^{* 2}\right)^{\frac{3}{2}} .
$$

where, $q=u, d, s$. The same expression is valid for the electrons with a factor $\frac{1}{3}$ in the right hand side.

The electromagnetic charge neutrality and the baryonic density are

$$
\begin{gathered}
2 n_{u}-n_{d}-n_{s}-3 n_{e}=0, \\
n_{B}=\frac{1}{3}\left(n_{u}+n_{d}+n_{s}\right),
\end{gathered}
$$

respectivelly. To solve the nonlinear system of Eqs. (5) and (6) we replace the quark densities $n_{q}$ by its corresponding expressions in terms of chemical potentials of (3).

The pressure of the $S Q M$ in the $D D Q M$ model aplying to the $S Q M$ case is given by the thermodynamic relation

$$
P=\left.\frac{\partial \Omega / n_{B}}{\partial\left(1 / n_{B}\right)}\right|_{T,\left\{\mu_{q}\right\}}=\left.n_{B} \frac{\partial \Omega}{\partial n_{B}}\right|_{T,\left\{\mu_{q}\right\}}-\Omega,
$$

where $\Omega$ is the thermodynamical potential of the system. Notice that the first term on the right hand side is necessary to make the pressure expression thermodynamically consistent with the energy density given by

$$
\varepsilon=\sum_{q} \varepsilon_{q}=\sum_{q}\left(-P_{q}+\mu_{q} n_{q}\right)-\left.T \frac{\partial \Omega}{\partial T}\right|_{n_{B},\left\{\mu_{q}\right\}},
$$

with the quark density defined by

$$
n_{q}=-\left.\frac{\partial \Omega_{q}}{\partial \mu_{q}}\right|_{T, n_{B}} .
$$

At $T=0$,the expressions for the quarks pressure and energy density are

$$
\begin{aligned}
P_{q} & =\frac{g_{q}}{48 \pi^{2}} m_{q}^{4}\left[F\left(x_{q}\right)-\frac{C}{n_{B}} \frac{4}{m_{q}} G\left(x_{q}\right)\right], \\
\varepsilon_{q} & =\frac{g_{q}}{48 \pi^{2}} m_{q}^{4}\left[3 H\left(x_{q}\right)+\frac{C}{n_{B}} \frac{4}{m_{q}} G\left(x_{q}\right)\right],
\end{aligned}
$$

where $x_{q}=\left[\left(\frac{\mu_{q}}{m_{q}^{*}}\right)^{2}-1\right]^{\frac{1}{2}}$.

In the above equations the auxiliary functions $F, G$ and $H$, are defined as

$$
\begin{gathered}
F(x)=x\left(x^{2}+1\right)^{1 / 2}\left(2 x^{2}-3\right)+3 \sinh ^{-1}(x), \\
G(x)=x\left(x^{2}+1\right)^{1 / 2}-\sinh ^{-1}(x),
\end{gathered}
$$

and

$$
H(x)=x\left(x^{2}+1\right)^{1 / 2}\left(1+2 x^{2}\right)-\sinh ^{-1}(x) .
$$

The contributions of free electrons to the thermodynamic potential is given by

$$
\Omega_{e}=-\frac{1}{12 \pi^{2}} \mu_{e}^{4}
$$

From Eq.(10) we can see that there is a given value of baryonic density for which the pressure is null. At this density the null pressure can be interpreted as a consequence of the attractive internal interaction among the constituents of the system, simulated by the change of $M_{D}$ in the model. This null pressure situation represents the quark matter confinement. On other hand in Eq.(10), the increase of density leads to a pressure expression of a free fermionic gas, characterizing the asymptotic freedom.

\section{THE $C F L$ PHASE}

In the $C F L$ phase the Cooper pairs occup the same lowest energy quantum state at zero temperature, leading to a Bose condensate. In this configuration, the ground state of quark matter becomes a color superconductor[6], [7], [8]. The main consequence of color superconductivity is the appearance of a nonzero energy gap in the one-particle energy, $\varepsilon_{i}=\sqrt{\left(E_{i}-\mu\right)^{2}+\Delta^{2}}$, with $\Delta$ being the CFL superconducting gap.

The $C F L$ phase is characterized by same density of quarks $u, d$ and $s$, so the charge neutrality is automatically satisfied. The gap $\Delta$ can be calculated with perturbative $Q C D$ with quark-gluon exchange[ref.], but in this work we consider it like a free parameter whit characteristic values for the transition.

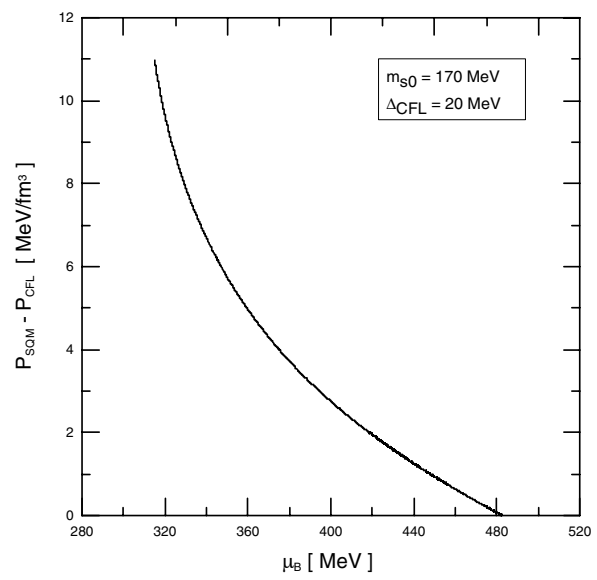

FIG. 1: Pressures difference, $P_{S Q M}-P_{C F L}$, versus baryonic chemical potential, $\mu_{B}$ for $m_{s 0}=170 \mathrm{MeV}$. The difference between the pressures is null for the critical value $\mu_{B} \sim 482 \mathrm{MeV}$, coinciding whit $\mu_{C}$ showed in the Fig.(2-a) in which the phase transition occurs. 

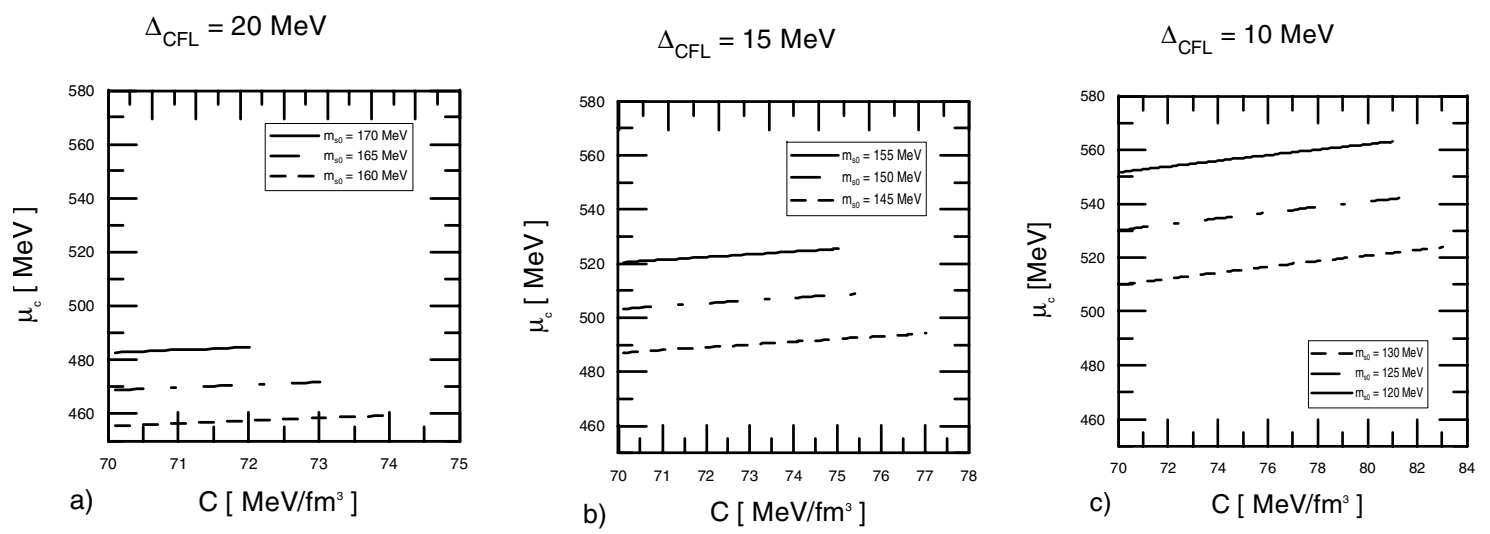

FIG. 2: Critical chemical potential, $\mu_{c}$, versus the $C$ parameter of the $D D Q M$ model for differents $m_{s 0}$, fixing the gap in all the cases: a) $\Delta_{C F L}=20 \mathrm{MeV}$, b) $\Delta_{C F L}=15 \mathrm{MeV}$ e $\Delta_{C F L}=10 \mathrm{MeV}$.

The pressure in the $C F L$ reads

$$
P_{C F L}=\sum_{q} P_{q}+\frac{3 \Delta^{2} \mu^{2}}{\pi^{2}},
$$

where the first term gives the pressure of free quarks, with equal number densities $n_{u}=n_{d}=n_{s}=\left(v^{3}+2 \Delta^{2} \mu\right) / \pi^{2}$, where $\mathrm{v}$ is the common Fermi momentum, given by

$$
v=2 \mu-\sqrt{\mu^{2}+\frac{m_{s}^{2}}{3}},
$$

and $\mu=\left(\mu_{u}+\mu_{d}+\mu_{s}\right) / 3$. The second term in Eq. (16) is the contribution from the formation of the condensate to the pressure.

\section{RESULTS AND CONCLUSIONS}

We have applied the Gibbs conditions for construct the phase transition between the two phases described in the last section. The critical chemical potential is defined as the value for which the pressure of the pure $S Q M$ phase equals the pressure of the pure $C F L$ phase. Besides the Gibbs conditions, the local (neutral) electromagnetic charge of each phase and the global baryonic charge conservation are simultaneously required. In Fig.(1) we can see the difference of pressures $P_{S Q M}-P_{C F L}$ as a function of the baryonic chemical potential, for a given value of the gap and current strange quark mass, $m_{s 0}$, showing the critical potential value.

The main result of this work consists in discuss the sensitivity of the critical chemical potential of the phase transition in the $D D Q M$ model parameter, $C$. In Fig.(2) we display the critical chemical potential as a function of the parameter $C$, for different values of the superconducting gap and $m_{s 0}$. The results show that the critical chemical potential is less sensitive to the model parameter $C$ than the gap and the current strange quark mass. Consequently, it is very important to have a satisfactory values for these last two external parameters in order to obtain a more accurate discution for the $S Q M-C F L$ phase transition.
[1] A. R. Bodmer, Phys. Rev. D 4, 1601 (1971).

[2] E. Witten, Phys. Rev. D 30, 272 (1984).

[3] M. Alford, K. Rajagopal, and F. Wilczek, Nucl. Phys. B 537, 443 (1999); R. Rapp, T. Schaefer, and T. Schaefer, Nucl. Phys. B 575, 269 (2000).

[4] G.N. Fowler, S. Raha, and R.M. Weiner, Z. Phys. C 9, 271
(1981).

[5] O. G. Benvenuto and G. Lugones, Phys. Rev. D 51, 1989 (1995).

[6] J. A. Bowers and K. Rajagopal, Phys. Rev. D 66, 065002 (2002).

[7] K. Rajagopal and F. Wilczek, Phys. Rev. Lett. 86, 3492 (2001).

[8] M. Alford and S. Reddy, Phys. Rev. D 67, 074024 (2003). 